\title{
Study on the Characteristic of Carrier Frequency- Iron Loss of Induction Motor Fed with Variable Frequency Power
}

\author{
Chengfang $\mathrm{Ji}^{1 \text {, a }}$, Juanping Zhao ${ }^{1, \mathrm{~b}}$ \\ ${ }^{1}$ School of Mechanical and Electrical Engineering, Zhengzhou University of Industry Technology, \\ Zhengzhou 450000, China. \\ ajichengfang8897@163.com, ${ }^{b}$ zjptiming@foxmail.com
}

Keywords: Carrier frequency, iron loss, induction motor, variable frequency power.

\begin{abstract}
Aiming at the problem of reducing consumption of asynchronous motor caused by carrier frequency in frequency conversion power supply, starting from the perspective of carrier frequency, through the study of the relationship between the iron loss and the carrier frequency,and using the finite element analysis software to establish the model of motor and MATLAB inverter power,which are carried out the joint simulation calculation. The characteristic of relationship between the iron loss of the motor and the carrier frequency proved by simulation when the carrier frequency inverter power supply, which has a reference value for the design of frequency conversion motor, frequency conversion applications and energy saving.
\end{abstract}

\section{Introduction}

In today's increasingly serious energy problems, it is important to improve the efficiency of induction motor not only from the viewpoint of energy saving, but also from the broad perspective of environmental.In the motor, the core loss is the constant loss, which accounts for a large proportion of the total loss.Especially with the development of power electronic technology, more and more motors are powered by inverter.The harmonic component of the inverter output will not only generate additional iron losses in the stator core, but also generate additional losses in the rotor core[1].Taking into account the motor rotor cooling conditions are relatively poor, which will affect the working life of the motor.Therefore, it is necessary to further analyze the core loss of variable frequency induction motor in order to improve the working performance.

\section{The Characteristics of Iron Loss of the Motor}

The iron loss are divided into magnetic hysteresis loss and eddy current loss.The ferromagnetic material in an alternating magnetic field, the domain will flip back and forth to make it with the external magnetic field in the same direction,resulting in loss of friction between each other, known as hysteresis loss. The hysteresis loss formula is:

$$
P_{\mathrm{h}}=V f C_{h} B_{m}^{k}
$$

In the formula: $C_{h}$-Hysteresis loss coefficient; $B_{m}$-The maximum magnetic flux density of hysteresis loop; $\mathrm{k}=1.6 \sim 2.3$.

When the magnetic flux in the core of the alternative, according to the law of electromagnetic induction, which will generate the induction electromotive force and current in the iron core.As the core is also a conductor, the current will flow around the magnetic flux in the core, known as the eddy currents. The loss produced in the iron core is called eddy current loss.Eddy current loss formula for

$$
P_{\mathrm{e}}=V f^{2} \pi^{2} \Delta^{2} B_{m}^{2} / 6 \rho
$$

In the formula: $\Delta$-The thickness of silicon steel sheet; $\rho$-The core resistivity.

The basic iron loss formula of the motor can be known as follows:

$$
P_{h}=V f C_{k} B_{m}{ }^{k}+V f^{2} \pi^{2} \Delta^{2} B_{m}^{2} / 6 \rho
$$

In engineering calculations, the commonly used iron loss formula is: 


$$
P_{\mathrm{Fe}}=C_{\mathrm{Fe}} f^{1.3} B_{m}^{2} G
$$

In the formula: $C_{F e}$-The iron loss coefficient; $G$-The core weight.

When the induction motor variable frequency power supply, the lower the carrier frequency, the greater the harmonic content of the current waveform. So that each harmonic can be superimposed together, the total effective value of the motor current will become larger, so that the increase in iron consumption.The larger the carrier frequency, the more close to the current waveform of the motor current waveform, the smaller the harmonic content, the increase in the amount of iron loss will be reduced.It can be seen that the motor iron loss is decreased with the increase of the carrier frequency.The relationship between the iron loss of the motor and the carrier frequency is shown in Figure 1[2, 3].

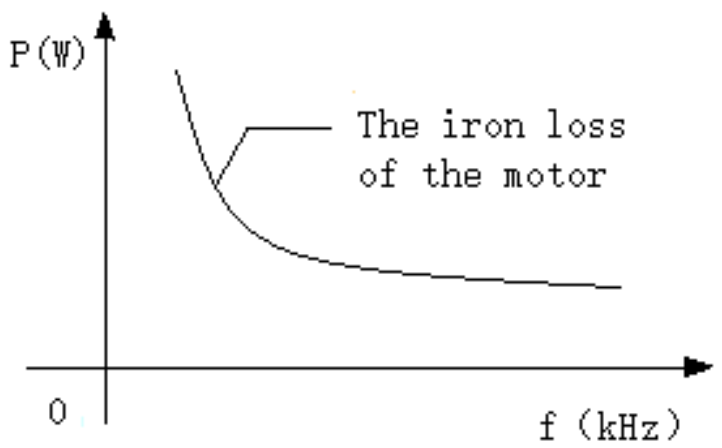

Fig. 1 The relation curve between the iron loss of the motor and carrier frequency

\section{The Simulation Analysis of Iron Loss}

Set up the Finite Element Model of the Motor. The model of the motor was established by using finite element analysis software in this article,which carried out electromagnetic simulation,the motor loss is obtained under the condition of power frequency operation. The motor entity model is set up in MagNet as shown in figure 2. Frequency conversion power model is set up in MATLAB/Simulink software. The joint simulation model is set up with MagNet software and MATLAB/Simulink software,then the simulation analysis was carried out when electric mechanic frequency conversion power supply. The joint simulation model as shown in figure $3[4,5]$.

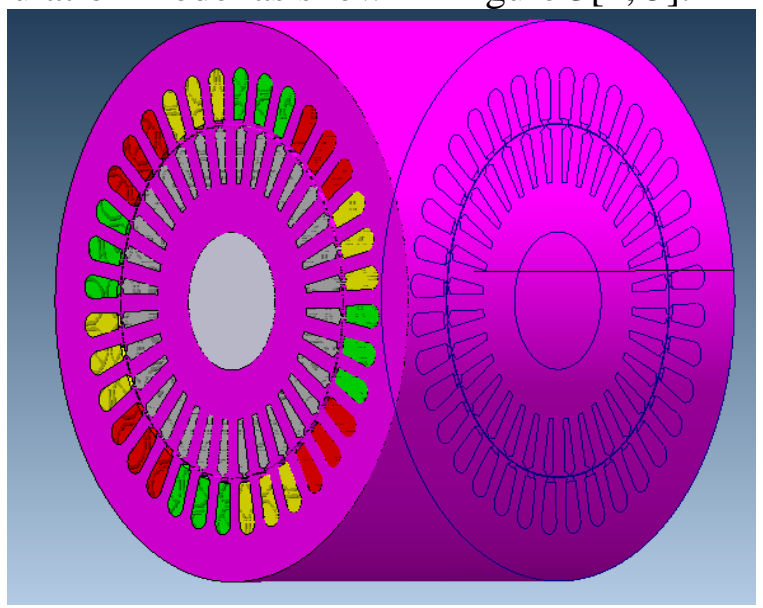

Fig.2 The entity model of the machine 


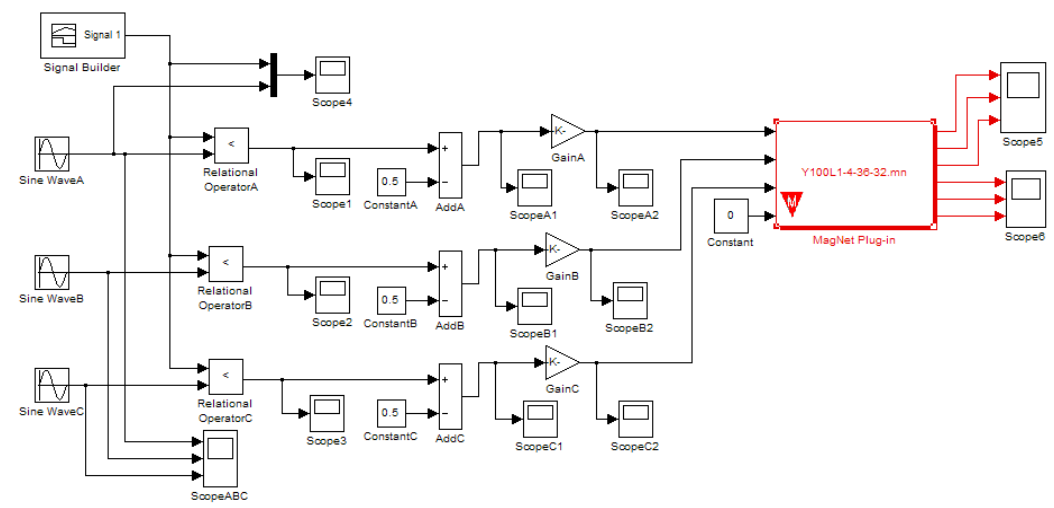

Fig. 3 The joint simulation model

Simulation Calculation Result Analysis. According to the simulation results, it can be concluded that,the waveform of the motor iron loss of power frequency power supply is shown in a simulation cycle, as shown in figure 4.It can be seen from the figure that the iron loss of the motor is periodic,so the iron loss of the motor is the average value of the period,Therefore, the average value of the iron loss in a stable period of power frequency power supply is $46.5 \mathrm{~W}$.

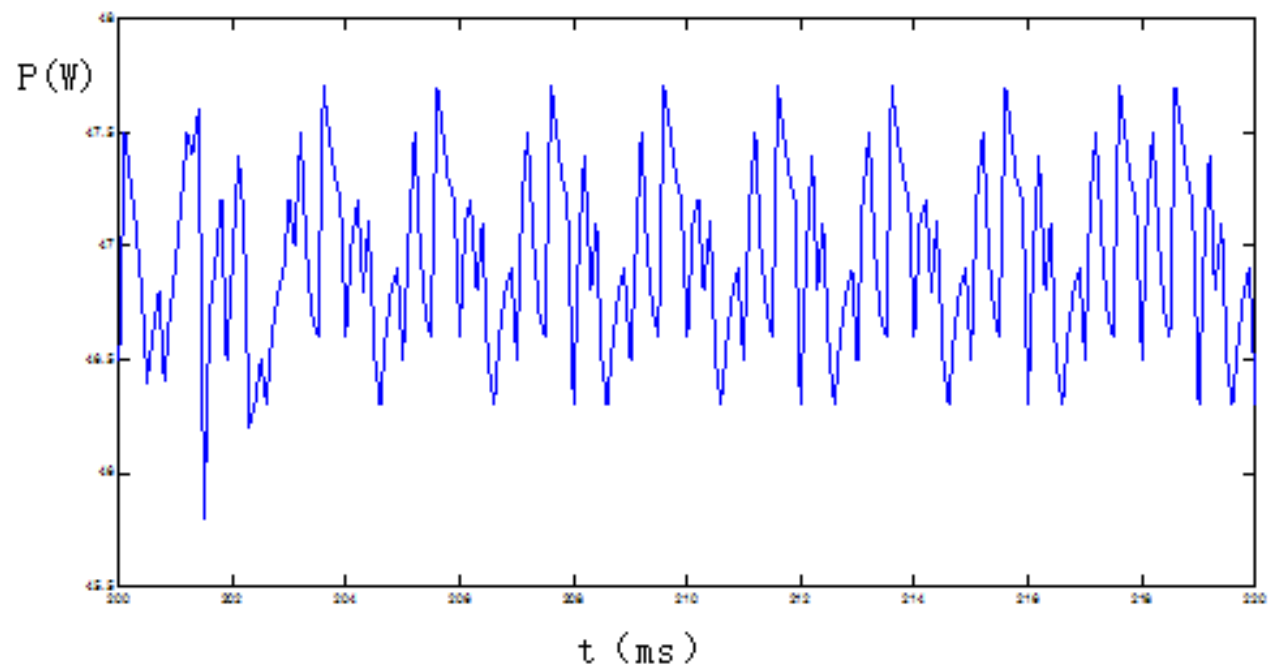

Fig.4 The waveform of iron loss in stable cycle under power frequency power supply

When the carrier frequency is $2 \mathrm{kHz}$, The waveform of the motor iron loss in a simulation period is shown in Figure 5.As can be seen from the figure, the average value of the iron loss of the motor in a stable period under variable frequency power supply is $52.42 \mathrm{~W}$.

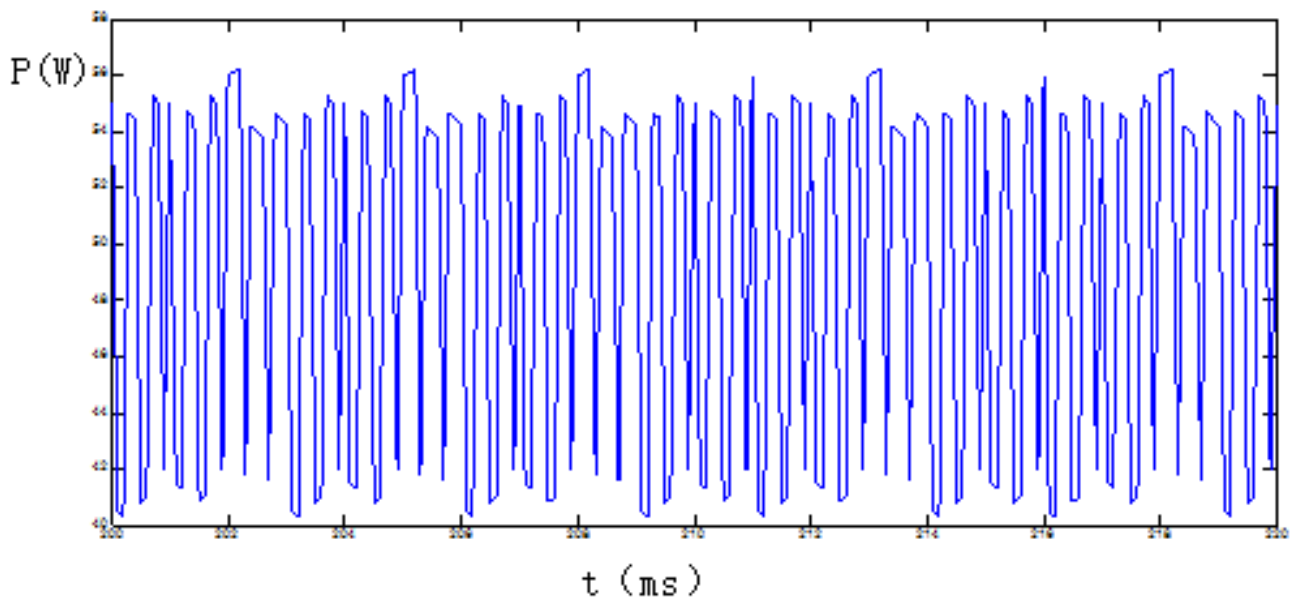

Fig.5 The waveform of iron loss in stable cycle under variable frequency power supply

When the carrier frequency is $5 \mathrm{kHz}$, The waveform of the motor iron loss in a simulation period is shown in Figure 6.As can be seen from the figure, the average value of the iron loss of the motor in a stable period under variable frequency power supply is $51.59 \mathrm{~W}$. 


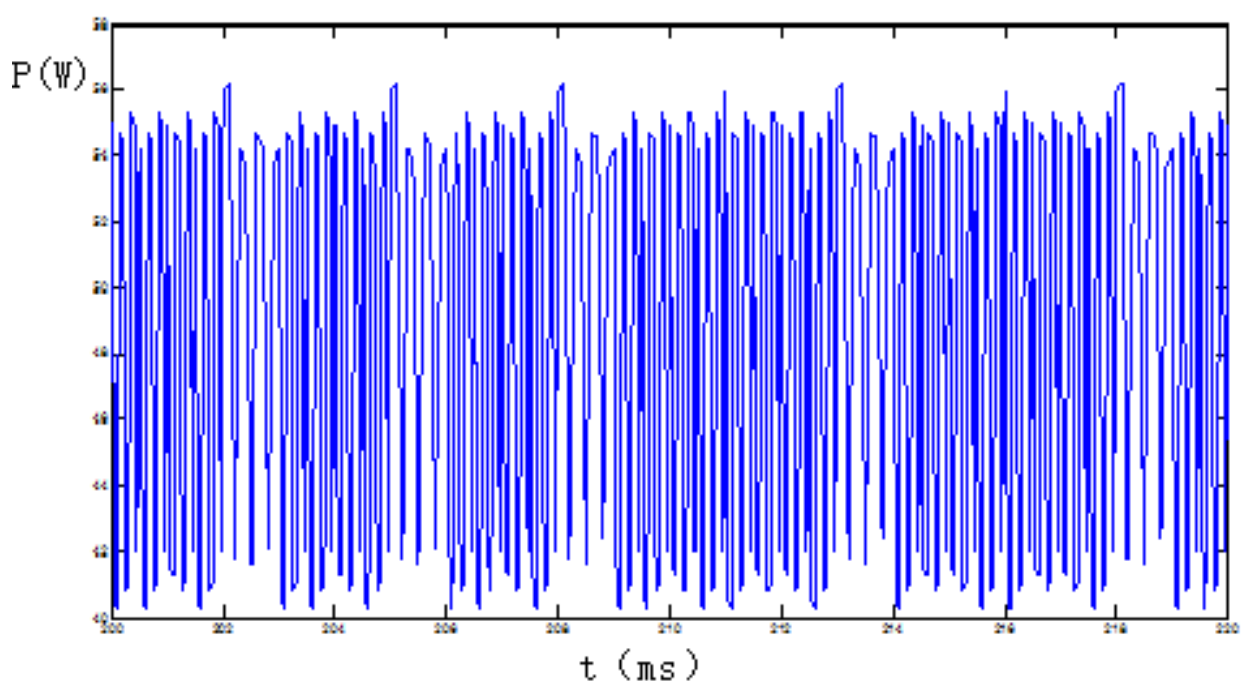

Fig.6 The waveform of iron loss in stable cycle under variable frequency power supply

Can be seen from the figure 4 and 5 , the iron loss of the motor under frequency conversion power supply is greater than under the power frequency power supply.Can be seen from the figure 5 and 6 , when the carrier frequency is $5 \mathrm{kHz}$, the motor iron consumption of the waveform in a simulation cycle than the carrier frequency of $2 \mathrm{kHz}$, the waveform is more dense, more volatile.

\section{Summary}

Through the analysis of theory and simulation results, it is concluded that the relationship between the carrier frequency and the iron loss of the motor under variable frequency power supply. The theory is consistent with the simulation results, which is a good guide for the research and analysis of the frequency conversion motor design and energy saving.

\section{References}

[1] Wang Jun. Effect of carrier frequency of frequency converter for motor operation[J]. Qilu Petrochemical Technology, (2011),39(2)104-106.

[2] National standards of the People's Republic of China. Inverter power supply cage induction motor design and performance guideline[M]. Beijing:China Standard Publishing House,(2007).

[3] National standards of the People's Republic of China. Inverter power supply cage induction motor design and performance guideline[M]. Beijing:China Standard Publishing House,(2008).

[4] Wu Yaohui, Liu Xiaomei,Ji Chengfang, Research on the Temperature Field of Special Linear Motor [C], Applied Mechanics and Materials,(2013), 273 (416-417)

[5] Luo Weidong,Huang Ke,Qiu Wangbiao. Frequency conversion speed range of analysis Y100L1-4 motor[J]. Journal of hubei university(Natural science edition),(2008),30 (3):260-262. 